\title{
An immersed boundary method for simulating compressible viscous flow in complex geometries
}

\author{
B. Jastrow \& F. Magagnato \\ Institute of Fluid Machinery, Karlsruhe Institute of Technology, Germany
}

\begin{abstract}
This paper presents an immersed boundary method combined with a wall-layer approach implemented into an established flow solver. In the outer flow field, the compressible Navier-Stokes equations are solved using an approximate Riemann Solver whereas simplified boundary-layer equations are solved near the wall. Turbulence is accounted for by the one-equation model of Spalart-Allmaras in the outer flow region and by a mixing length eddy viscosity model with near wall damping in the wall layer. Computations performed for various test cases show good agreement with reference data found in literature.
\end{abstract}

Keywords: immersed boundary method, wall-layer model, compressible flow.

\section{Introduction}

Body-fitted grid generation for simulating flow in complex geometry can be very cumbersome especially when using a block-structured solver. Therefore the Cartesian-grid immersed boundary method (IBM) offers an interesting approach since automatic mesh generation can be realized easily. Furthermore, due to smoothness and orthogonality, Cartesian grids offer high accuracy and efficiency.

In the IBM a complex geometry is immersed into a regular Cartesian grid. The effect of the body on the flow is mimicked by the imposition of proper boundary conditions that act as forcing conditions [1,2].

Several applications of the IBM use a linear interpolation for setting the boundary conditions as proposed in [1]. Their validity is therefore only given for grids resolved down to $\Delta y^{+}<1$. For flow of high Reynolds number these restrictions cannot be held in accordance with acceptable computing time. With 
the use of a wall-layer model one can overcome the need for high near wall resolution. Wall models based on incompressible turbulent boundary layer equations were proposed and tested by Balaras et al. [3], Cabot and Moin [4], Wang and Moin [5] in a body-fitted context. The applicability of such a walllayer model in the framework of the IBM has been studied by Tessicini et al. [6]. Bond and Blottner [7] developed a compressible wall model, named the diffusion model.

The present paper studies the compressible wall-layer model within the framework of the IBM. It is shown, that the implementation in the in-house flow solver SPARC (Structured Parallel Research Code) [8], in the following referred to as SPARC-IBM, provides results for basic test cases in good agreement with literature.

\section{Mesh generation}

The mesh is automatically generated via a ray tracing technique. Based on a geometry described by a closed surface triangulation every cell center of a uniform Cartesian grid is marked as either internal or external [9]. Those internal cells having at least one external neighbor cell are marked as wall-layer cells. From the latter the normal to the closest wall is computed and stored together with the forcing point and the appropriate interpolation neighbors (figure 1). The current implementation of the mesh generator uses a block-refinement algorithm based on the wall distance.

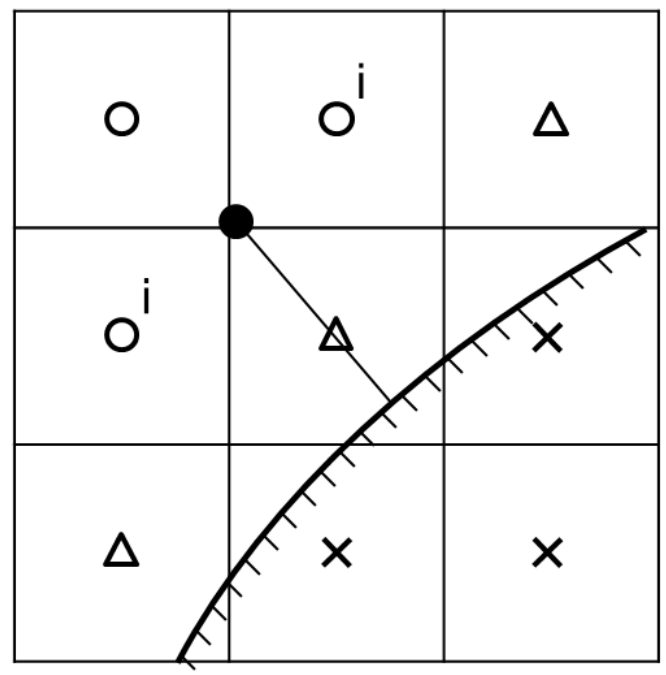

Figure 1: Mesh in the near wall region: $\circ$ : internal cell, $\Delta$ : wall-layer cell, $\mathrm{x}$ : external cell, $\bullet$ : forcing point, i: interpolation neighbors. 


\section{Numerical method}

The Reynolds-averaged Navier-Stokes (RANS) equations for compressible flow can be written as follows:

$$
\begin{gathered}
\frac{\partial \rho}{\partial t}+\frac{\partial\left(\rho u_{j}\right)}{\partial x_{j}}=0 \\
\frac{\partial\left(\rho u_{i}\right)}{\partial t}+\frac{\partial\left(\rho u_{i} u_{j}+p \delta_{i j}-\tau_{i j}\right)}{\partial x_{j}}=0 \\
\frac{\partial\left(\rho e_{t}\right)}{\partial t}+\frac{\partial\left(\rho u_{j} e_{t}+p u_{j}-u_{i} \tau_{i j}+q_{j}\right)}{\partial x_{j}}=0 .
\end{gathered}
$$

For the calculations carried out in this paper, the Spalart-Allmaras turbulence model has been used for closure. Details of the model are given in the paper by Spalart and Allmaras [10]. The discretization of the convective terms is obtained by an approximate Riemann solver (HLLC) [11] whereas the viscous terms are discretized with a central difference scheme. The solution vector is updated via a Runge-Kutta scheme.

Inside the wall-layer a simplified set of equations is solved. It is assumed that convection is negligible compared to diffusion, the normal pressure gradient is zero and the streamwise gradients are of orders lower than the gradients normal to the wall. Furthermore, the normal velocity is insignificant compared to the tangential velocity. This leads to the following equations for the $\mathrm{x}$-momentum and the energy:

$$
\begin{gathered}
\frac{\partial(\rho u)}{\partial t}=\frac{\partial\left[\left(\mu+\mu_{t}\right) \frac{\partial u}{\partial y}-y \frac{\partial p}{\partial x}\right]}{\partial y} \\
\frac{\partial\left(\rho e_{t}\right)}{\partial t}=\frac{\partial\left[u\left(\mu+\mu_{t}\right) \frac{\partial u}{\partial y}+\left(\kappa+\kappa_{t}\right) \frac{\partial T}{\partial y}\right]}{\partial y} .
\end{gathered}
$$

The properties $\mu_{t}$ and $\kappa_{t}$ are the turbulent viscosity and the turbulent conductivity respectively. In a first stage of implementation, $\mu_{t}$ is obtained by a mixing length eddy viscosity model with near wall damping

$$
\frac{\mu_{t}}{\mu}=\kappa y^{+}\left(1-e^{-\frac{y^{+}}{A}}\right)^{2},
$$

where $\kappa=0.4, A=19$ and $y^{+}$defines the dimensionless distance to the wall [5]. At the wall, a no-slip boundary condition is applied. At the forcing point, that defines the outer edge of the wall-layer, the boundary condition is obtained through an interpolation from the flow values of the outer flow field. In this paper, the equations are further simplified by neglecting the time derivative terms and the streamwise pressure gradient. Two tridiagonal systems are created for the velocity and the temperature respectively and solved in a segregated way with the other dependent variables held constant. Subsequently the turbulent viscosity is updated. An outer loop runs over all equations until the full solution converges. Finally the flow values for the position of the center of the wall-layer cell is extracted and provided to the outer flow field as a boundary condition.

Assuming steady-state in the wall-layer corresponds to an instantaneous response of the wall-layer to the outer flow field which creates some error for unsteady flow calculations. 


\section{Results}

\subsection{Laminar flow past a flat plate}

The first test case is the laminar flow past a flat plate. Since the wall-layer model also captures the linear near-wall behavior for low Reynolds number flow, this test case is perfectly suited for verifying the implementation. The computation was carried out on a structured multi-block mesh with refinement. The boundary layer was resolved with 32 cells making a total of 54,000 cells in the 2D plane.

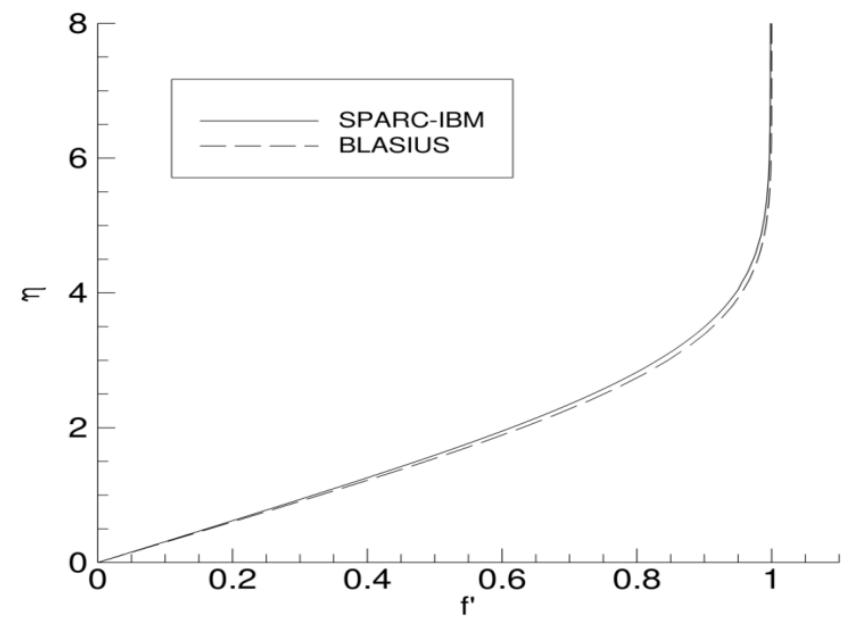

Figure 2: Comparison of the velocity profile in the boundary layer at $R e_{x}=10,000 R e_{x}=10,000$.

The Mach number was chosen to $M a_{\infty}=0.3$ and the velocity profile was extracted at a Reynolds number of $R e_{x}=10,000$. In Figure 2 the profile is compared with the analytical results of the Blasius-equation [12] where $f^{\prime}$ is the dimensionless velocity and $\eta$ is the dimensionless wall distance, defined as

$$
\begin{gathered}
f^{\prime}=\frac{U}{U_{\infty}} \\
\eta=y \sqrt{\frac{U_{\infty}}{v x}} .
\end{gathered}
$$

The numerical results show very good agreement with the analytic solution of Blasius.

\subsection{Turbulent flow past a flat plate}

The second test case was chosen to prove the consideration of turbulence in the wall-layer model. The mesh consists of 123,000 cells in the $2 \mathrm{D}$ plane which results in 27 cells resolving the boundary layer. Figures 3 and 4 show the 
obtained velocity profile for $R e_{x}=10,000,000$ and the skin friction coefficient along the flat plate together with the experimental data of [13]. The agreement is quite satisfactory.

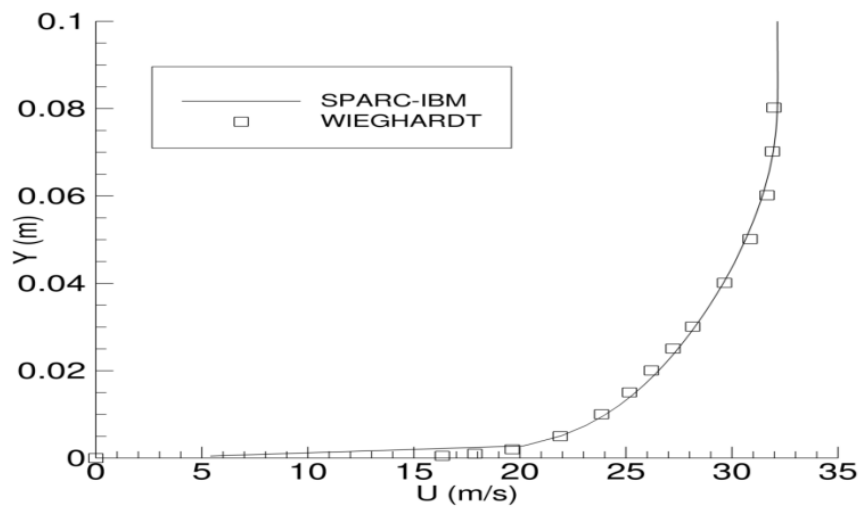

Figure 3: Comparison of the velocity profile in the boundary layer at $\operatorname{Re}_{x}=10,000,000$.

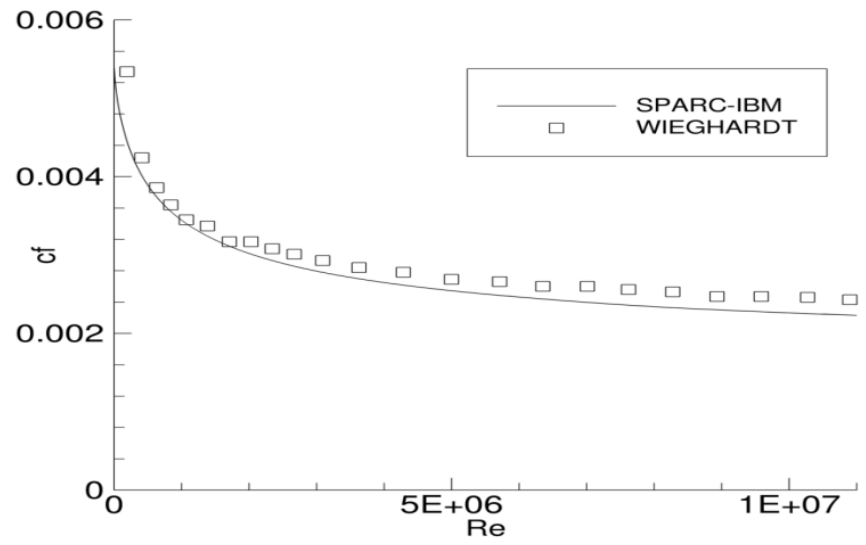

Figure 4: Comparison of the skin friction coefficient for various Reynolds numbers.

\subsection{Laminar flow past a circular cylinder}

To test the IBM together with curved surfaces, the flow past a circular cylinder was computed. Two flow regimes are shown, a steady flow at $R e=20$ and an unsteady flow at $R e=200$. A local view of the mesh is provided in Figure 5 . 


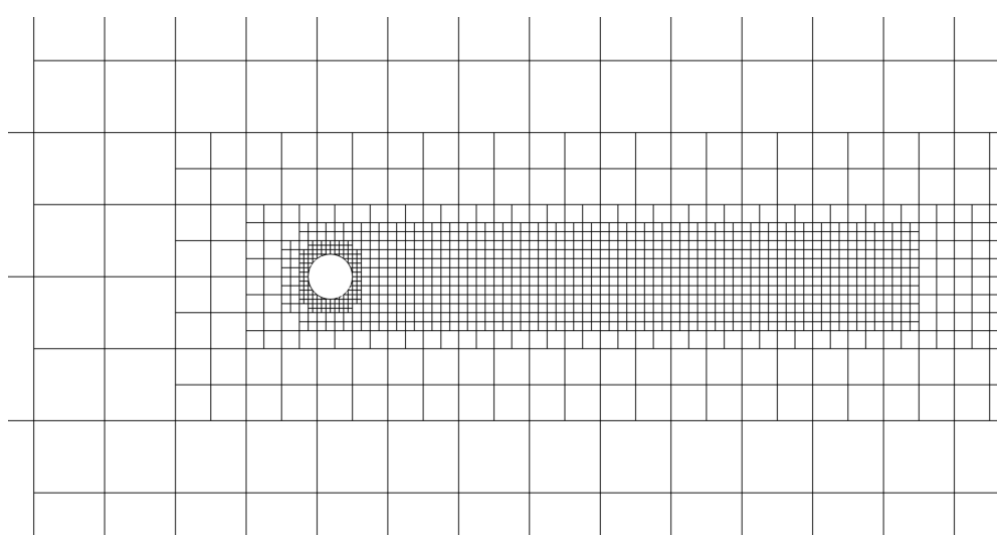

Figure 5: Local view of the mesh. Every eighth cell is plotted.

The wake region has been refined manually since a flow adaptive mesh refinement is subject to future work. The mesh consists of 105,000 cells in the 2D plane. The free stream Mach number in both cases was set to $M a_{\infty}=0.3$. The streamlines for the flow at $R e=20$ are shown in Figure 6 whereas Table 1 lists the separation length and angle and the drag coefficient in comparison with reference values from literature $[14,15]$. The agreement is quite satisfactory, only the separation angle is predicted too low, which might be due to the lack of considering the pressure gradient in the wall-layer.

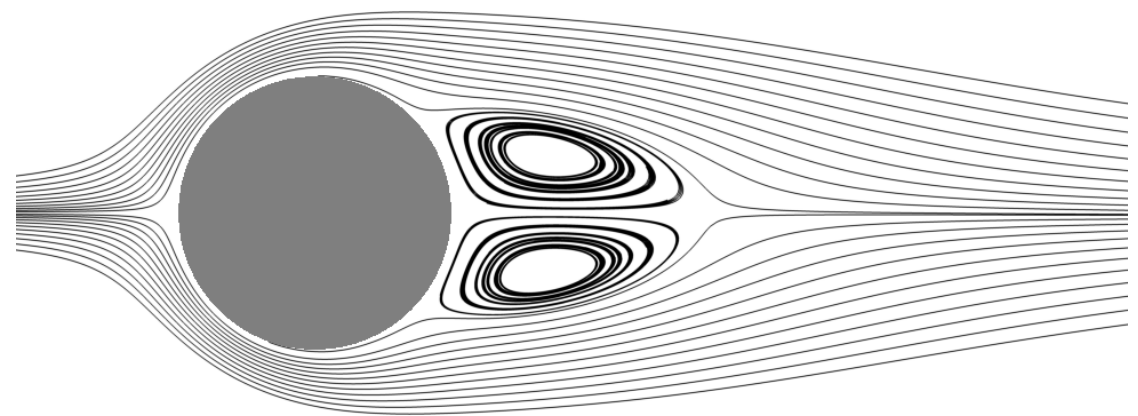

Figure 6: $\quad$ Streamlines for $R e=20$. 
Table 1: $\quad$ Separation length $L$, separation angle $\theta_{S}$ and drag coefficient $c_{D}$ for $R e=20$.

\begin{tabular}{c|c|c|c}
\hline & $L$ & $\theta_{S}$ & $c_{D}$ \\
\hline Sucker and Brauer [14] & 0.83 & $43.3^{\circ}$ & 2.02 \\
\hline Dennis and Chang [15] & 0.94 & $43.7^{\circ}$ & 2.05 \\
\hline SPARC-IBM & 0.93 & $41.8^{\circ}$ & 2.09 \\
\hline
\end{tabular}

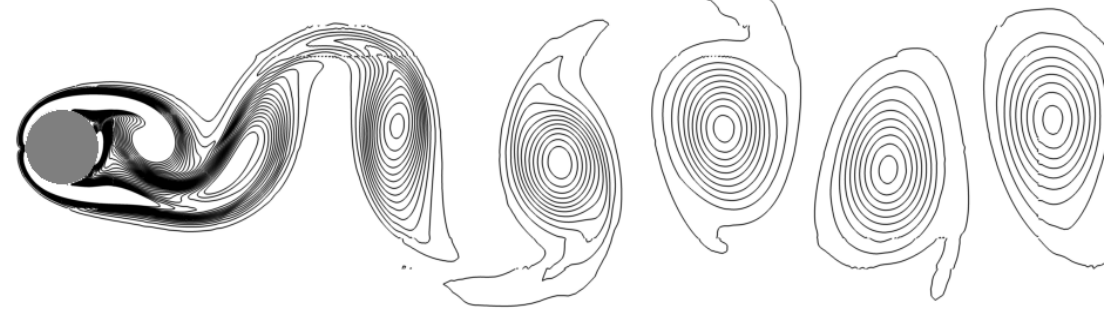

Figure 7: Instantaneous contours of the vorticity for $R e=200$.

Figure 7 shows the instantaneous contours of the vorticity for an unsteady laminar flow around the cylinder at $R e=200$. A comparison of the Strouhal number and the mean drag coefficient with results from literature [16, 17] is provided in Table 2 .

Table 2: $\quad$ Strouhal number $S t$ and mean drag coefficient $c_{D}$ for $R e=200$.

\begin{tabular}{c|c|c}
\hline & $S t$ & $c_{D}$ \\
\hline Linnick and Fasel [16] & 0.197 & 1.34 \\
\hline Liu et al. [17] & 0.192 & 1.31 \\
\hline SPARC-IBM & 0.193 & 1.38 \\
\hline
\end{tabular}




\subsection{Turbulent flow past a circular cylinder}

The unsteady turbulent flow around a circular cylinder at $R e=140,000$ is provided as a further example. The mesh is taken from the previous stated calculations and the free stream Mach number was set to $M a_{\infty}=0.3$. Figure 8 shows the time averaged pressure distribution in terms of the pressure coefficient $c_{p}$ in comparison with experimental results from Cantwell and Coles [18]. The angle $\alpha$ refers to the position on the surface of the cylinder beginning at the stagnation point. The calculation cannot predict the pressure distribution well.

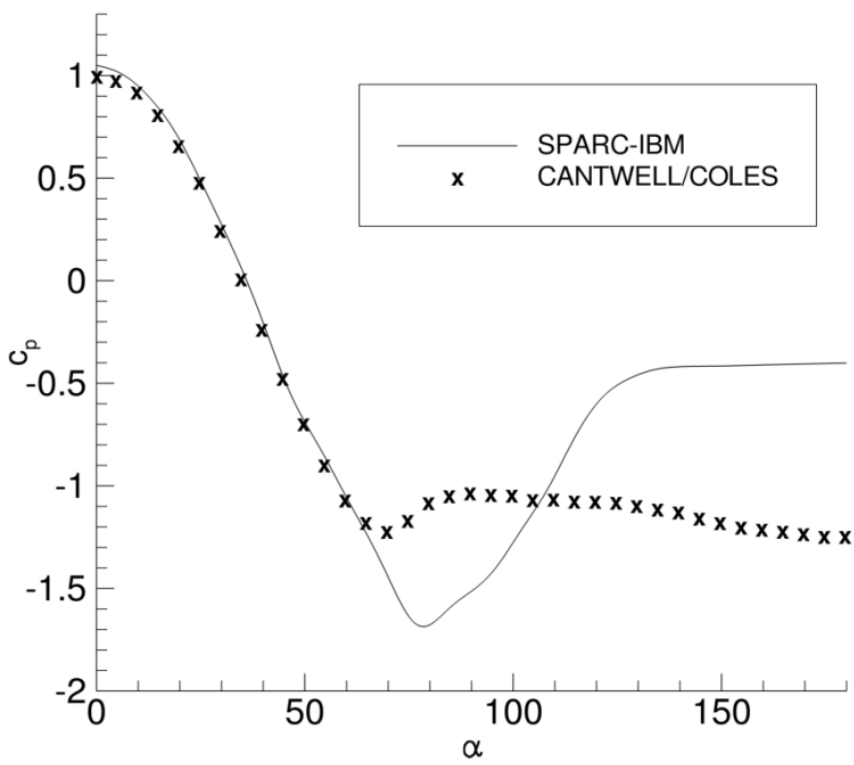

Figure 8: $\quad$ Pressure distribution at $R e=140,000$.

The minimum $c_{p}$ is about $40 \%$ lower than the measured value. In the separation area the pressure coefficient is determined too high resulting in a total drag coefficient of $c_{D}=0.52$ which is much lower than the measured value of $c_{D}=1.24$. Differences are also obtained for the Strouhal number. The computed value of $S t=0.23$ is much higher than the measured value of $S t=0.18$. The discrepancies are mostly due to the lack of the RANS turbulence model to predict the separation point correctly. Furthermore, turbulence is treated as isotropic which does not reflect reality. 


\section{Post processing}

Plotting the flow field obtained by SPARC-IBM requires an additional post processing since there exists no information about a body-fitted shape of the wall-layer cells. Therefore the boundary knots of every wall-layer cell are moved onto the closest wall via a ray tracing technique similar to the one used for the mesh generation. Figure 9 shows a section of the mesh around a cylinder with and without the additional post processing. The appropriate flow values are obtained by an inverse distance interpolation or from the wall values directly if applicable.
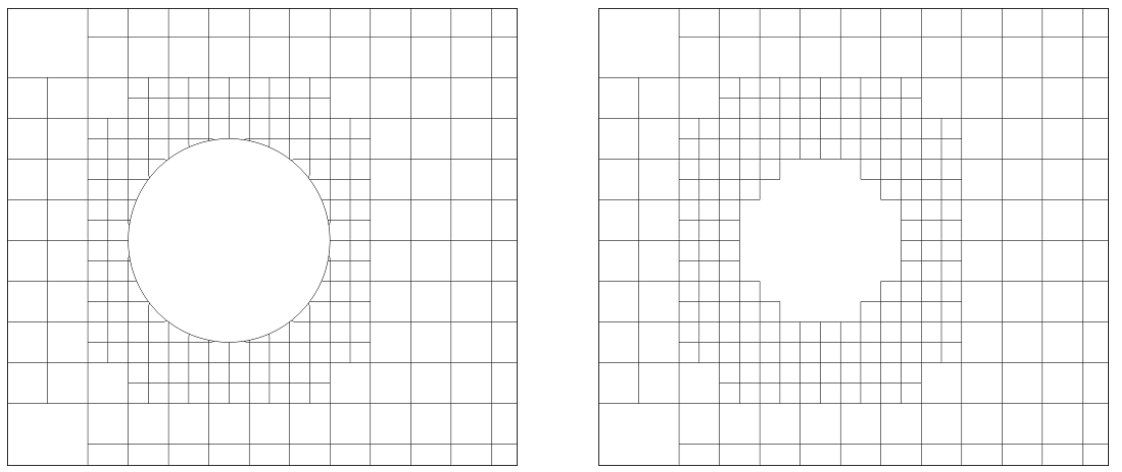

Figure 9: Local view of the mesh around a circular cylinder with (left) and without (right) the additional IBM-post processing. Every eighth cell is plotted.

\section{Conclusions and ongoing work}

The immersed boundary method (IBM) with a wall-layer approach has been implemented into an established block-structured code for solving compressible flow. The computation of basic test cases for laminar flow showed very good agreement with results found in literature. Viscosity dominated turbulent flow like the flow past a flat plate was also captured well. Discrepancies were obtained for turbulent flow with separation exemplarily shown for the flow past a circular cylinder. However, this is due to the well-known problem of turbulence modeling of bluff bodies rather than to the IBM.

To overcome the lack of consistency, future work comprises the testing of the simplified Spalart-Allmaras turbulence model for the wall-layer. It is believed that in contrast to using the algebraic model, the therewith associated full coupling of the outer flow field and the wall-layer provides better results for turbulent flows. Furthermore, the streamwise pressure gradient in the wall-layer and a flow adapted mesh refinement is about to be implemented. 


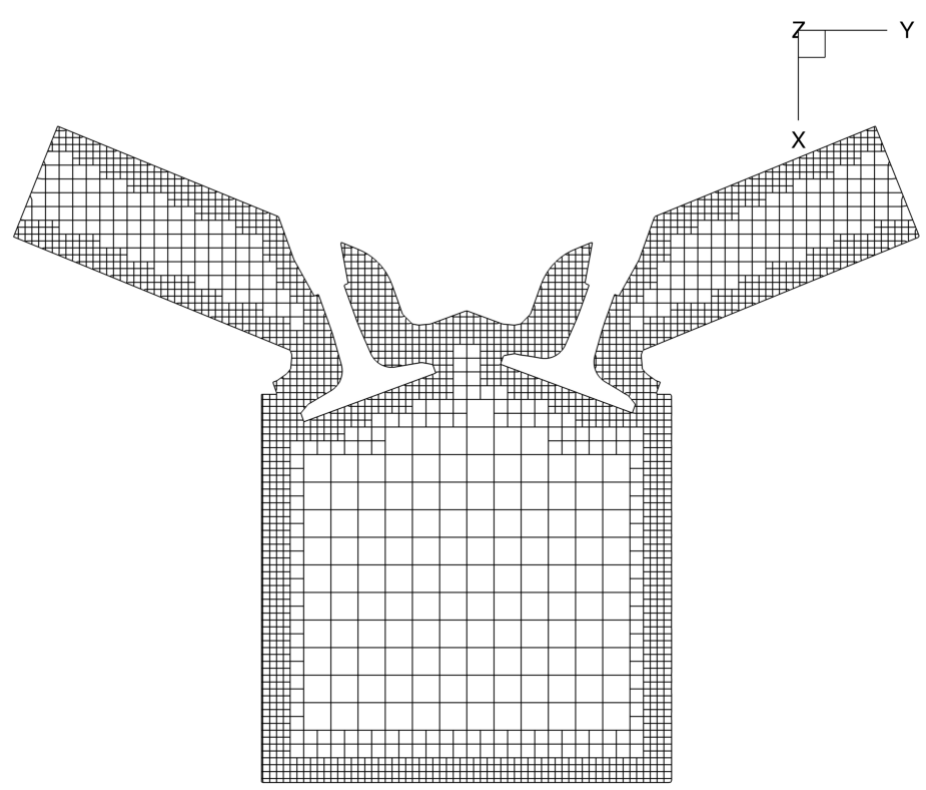

Figure 10: Slice through the IBM-mesh of an IC-engine. Every eighth cell is plotted.

Since the advantages of the IBM are only given for the simulation of complex geometries where the time for mesh generation is significant, the next step is to validate the implementation for $3 \mathrm{D}$ cases. As an example for a complex mesh a slice through the mesh of an internal combustion engine is shown in figure 10 .

\section{References}

[1] Fadlun, E.A., Verzicco, R., Orlandi, P. and Mohd-Yusof, J., Combined immersed-boundary finite difference methods for three-dimensional complex flow simulations. J. Comp. Phys., 161, pp. 35-60, 2000.

[2] Mohd-Yosuf, J., Combined immersed-boundary/B-spline methods for Simulations of flow in complex geometries. Annual Research Briefs, Center of Turbulence Research, pp. 317-328, 1997.

[3] Balaras, E. et al. Two-layer approximate boundary conditions for largeeddy simulations. AIAA Journal, 34, pp. 1111-1119, 1996.

[4] Cabot, W., Moin, P., Approximate wall boundary conditions in the largeeddy simulation of high Reynolds number flow. Flow, Turbulence and Combustion, 63, pp. 269-291, 1999.

[5] Wang, M., Moin, P., Dynamic wall modeling for LES of complex turbulent flows. Physics of Fluids, 14, pp. 2043-2051, 2002. 
[6] Tessicini, F., Iaccarino, G., Fatica, M., Wang, M., Verzicco, R., Wall modeling for large-eddy simulation using an immersed boundary method. Annual Research Briefs, Center of Turbulence Research, pp. 181-187, 2002.

[7] Bond, R.B., Blottner, F.G., Derivation, implementation, and initial testing of a compressible wall-layer model. Int. J. Numer. Meth. Fluids, 66, pp. 1183-1206, 2011.

[8] Magagnato, F., KAPPA - Karlsruhe Parallel Program for Aerodynamics, TASK Quarterly Vol.2, 2, pp. 215-270, 1998.

[9] Verzicco, R., Iaccarino, G., Immersed Boundary Technique for LargeEddy-Simulation. Lecture series on "Large Eddy Simulation and related techniques: Theory and Applications", 2006.

[10] Spalart, P.R., Allmaras, S.R., A one-equation turbulence model for aerodynamic flows. La Recherche Aerospatiale, 1, pp. 5-21, 1994.

[11] Toro, E.F., Spruce, M., Speares, M., Restoration of the contact surface in the HLL-Riemann solver, Shock Waves, 4, pp. 25-34, 1994.

[12] Blasius, H., Grenzschichten in Flüssigkeiten mit kleiner Reibung. Z. Math. Physik, 56, pp. 1-37, 1908.

[13] Wieghardt, K., Tillman, W., On the Turbulent Friction Layer for Rising Pressure. NACA TM-1314, 1951.

[14] Sucker, D., Brauer, H., Fluiddynamik bei quer angeströmten Zylindern. Wärme- und Stoffübertragung, 8, pp. 149-158, 1975.

[15] Dennis, S.C.R., Chang, G.Z., Numerical solutions for steady flow past a circular cylinder at Reynolds numbers up to 100. Journal of Fluid Mechanics, 42, pp. 471-489, 1970.

[16] Linnick, M.N., Fasel, H.F., A high-order immersed boundary method for unsteady incompressible flow calculations. J. Comp. Phys., 204, pp. 157192, 2005.

[17] Liu, C., Zheng, X., Sung, C.H., Preconditioned Multigrid Methods for Unsteady Incompressible Flows. J. Comp. Phys., 139, pp. 35-57, 1998.

[18] Cantwell, B., Coles, D., An experimental study of entrainment and transport in the turbulent near wake of a circular cylinder. J. Fluid Mech., 136, pp. 321-374, 1983. 